\title{
Myogenic potentials generated by a click-evoked vestibulocollic reflex
}

\author{
J G Colebatch, G M Halmagyi, N F Skuse
}

\begin{abstract}
Electromyograms (EMGs) were recorded from surface electrodes over the sternomastoid muscles and averaged in response to brief $(0 \cdot 1 \mathrm{~ms})$ clicks played through headphones. In normal subjects, clicks 85 to $100 \mathrm{~dB}$ above our reference (45 dB SPL: close to perceptual threshold for normal subjects for such clicks) evoked reproducible changes in the averaged EMG beginning at a mean latency of $8.2 \mathrm{~ms}$. The earliest potential change, a biphasic positive-negativity (p13-n23), occurred in all subjects and the response recorded from over the muscle on each side was predominantly generated by afferents originating from the ipsilateral ear. Later potentials (n34, p44), present in most but not all subjects, were generated bilaterally after unilateral ear stimulation. The amplitude of the averaged responses increased in direct proportion to the mean level of tonic muscle activation during the recording period. The p13-n23 response was abolished in patients who had undergone selective section of the vestibular nerve but was preserved in subjects with severe sensorineural hearing loss. It is proposed that the p13-n23 response is generated by activation of vestibular afferents, possibly those arising from the saccule, and transmitted via a rapidly conducting oligosynaptic pathway to anterior neck muscles. Conversely, the n34 and p44 potentials do not depend on the integrity of the vestibular nerve and probably originate from cochlear afferents.
\end{abstract}

$(F$ Neurol Neurosurg Psychiatry 1994;57:190-197)

Neurology, Institute of

Prince of Wales

Hospital, Randwick,

Sydney 2031, Australia

J E Colebatch

N F Skuse

Department of Neurology, Royal

Prince Alfred

Hospital,

Camperdown, Sydney

2050, Australia

G M Halmagyi

Correspondence to: Dr J G Colebatch,

Department of Neurology,

Institute of Neurological

Sciences, Prince of Wales

Hospital, High Street,

Randwick, Sydney 2031,

Australia

Received 18 December

1992 and in revised form

1992 and in revised
31 March 1993.

Accepted 8 April 1993 fall depends on otolith activation, possibly via connections to the reticular formation and thereby to the spinal cord. ${ }^{4-6}$ Detailed studies on a single patient who had an otolithic Tullio phenomenon (sound-evoked activation of the vestibular apparatus) showed shortlatency activation of leg muscles, probably via vestibulospinal pathways. ${ }^{7}$ The limitations of our knowledge of vestibular influences on the muscles of the trunk and limbs led us to reinvestigate earlier reports of activation of the vestibular apparatus in normal subjects by loud clicks.

Bickford et al ${ }^{8}$ described the characteristics of averaged responses to clicks with recordings with an active electrode just below the inion (the "inion response"). They concluded that the short latency potentials that they recorded were not, as they had first supposed, indicative of an auditory projection to the cerebellar vermis, but rather were generated by reflex changes in the level of EMG of posterior neck muscles, and thus were "myogenic" in origin. They also reported that the evoked response was present in patients with sensorineural deafness, leading them to propose that it arose from activation of the vestibular apparatus and not from activation of the cochlea. Subsequent publications by these workers described the inion response in patients with different acoustic and vestibular lesions and provided further evidence suggesting that it depended on activation of the otoliths, specifically, the saccule. ${ }^{910}$ The role of the vestibular apparatus has been disputed by others. Meier-Ewert et al ${ }^{11}$ argued that the inion response was a part of a generalised sonomotor response that originated from cochlear afferents. Additionally, averaged responses at the inion were not unique to click stimuli: Cody and Bickford ${ }^{12}$ were able to record a similar-looking response to both light flashes and electric shocks, albeit at longer latency than the response to sound. These uncertainties have led to the view that the inion response is non-specific and it has not been accepted as a useful measure of vestibular reflex function. ${ }^{13}$

We have used different recording sites from those of Bickford and co-workers, to demonstrate the presence of short latency EMG changes in anterior neck muscles after clicks. Also, the response can be separated into two distinct components: only the earlier of these depends specifically on the integrity of vestibular afferents. The findings from one patient have been the subject of a brief report. ${ }^{14}$ 


\section{Methods}

Recordings were made from 10 normal volunteers (seven men, three women; ages 29 to 63 years) and from eight patients with well defined lesions affecting the inner ear. For most of the recordings, the subjects were seated with a padded bar adjusted to be just in front of their foreheads. The subjects pressed against the bar to activate their neck flexors to the degree required throughout the averaging runs (see later).

Surface EMG activity was recorded from symmetrical sites over the upper half of each sternomastoid muscle by means of surface electrodes with a reference over the upper sternum. These sites were chosen in preference to the inion both to allow greater certainty as to the specific muscles likely to be generating any response seen, and also to avoid the uncertainties inevitably associated with the use of a midline recording site when investigating the effects of unilateral stimuli. The EMG from each side was amplified, bandpass filtered ( $8 \mathrm{~Hz}-1.6 \mathrm{kHz})$, and averaged using a sampling rate for each channel of $2.5 \mathrm{kHz}$. Initial experiments were performed with a Medelec Sensor evoked potential averager (Medelec Ltd, Surrey, UK); later experiments used a laboratory interface (1401, CED Electronics Ltd, Cambridge, UK) and an IBM compatible personal computer. Simultaneous averages of unrectified and rectified EMG were collected from $20 \mathrm{~ms}$ before the clicks to $80 \mathrm{~ms}$ afterwards. The clicks were presented at $3 / \mathrm{s}$ (preliminary findings indicated little change in the responses with repetition rates up to $5 / \mathrm{s}$ ) and the response to 512 stimuli averaged for each condition. Also, EMG activity was rectified and low pass filtered for display on an oscilloscope screen in front of the subject. A target level of EMG activity was set and the subjects instructed to keep the levels of smoothed, rectified EMG just above the target level for the duration of each average (about three minutes). The usual target corresponded to a mean rectified EMG activity of about
Subject 1

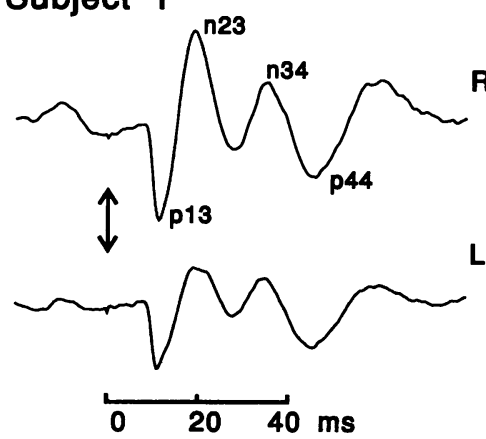

\section{Subject 2}

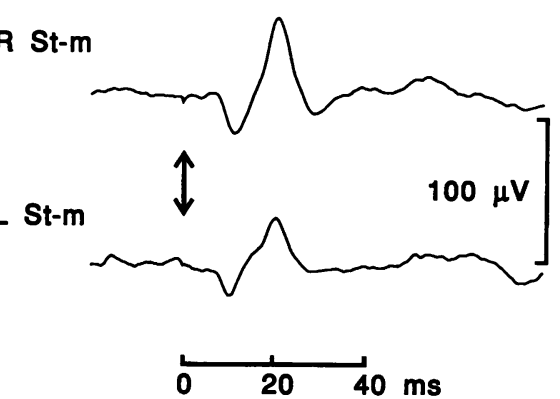

Figure 1 Averaged unrectified EMG responses recorded from surface electrodes over the right $(R S t-m)$ and left $(L S t-m)$ sternomastoid muscles in two different normal subjects. In this and subsequent illustrations, the unrectified averages are shown such that negative voltage changes at the active electrode cause an upward deflection. Both sets of averages (each of 512 trials) were obtained with the same click duration and intensity ( $95 \mathrm{~dB}$ above the reference intensity, see methods) and during tonic activation of the neck muscles. The clicks were applied $20 \mathrm{~ms}$ after the beginning of the sweeps, at the time shown by the arrows. In each subject the responses on the right and left sides were similar. The earliest potential, a diphasic positive-negativity ( $p 13-n 23)$, was present in both the subjects as well as all the other normal volunteers. In most subjects this was followed by additional potentials (n34, p44), as shown for subject 1.
50-60 $\mu \mathrm{V}$. In most trials the subjects were told to press their foreheads against the bar in front of them to achieve the necessary bilateral muscle activation but recordings were also made while the subjects held their heads turned to one side (predominantly unilateral muscle activation) and during bilateral muscle activation due to voluntarily holding the head slightly raised when lying supine.

The clicks were rarefactive square waves $0.1 \mathrm{~ms}$ long, generated by an ST10 evoked potential stimulator (Medelec Ltd, Surrey, UK) and delivered by calibrated headphones. Click intensities were adjustable in $5 \mathrm{~dB}$ increments. Although the subjects' threshold for detecting the clicks was always measured, the click intensity actually used was not related to their subjective thresholds and the same stimulus intensity was always used for both ears. With the duration used here $(0.1 \mathrm{~ms})$, most subjects cannot hear clicks softer than $45 \mathrm{~dB}$ SPL, and this level was therefore used as a reference (the same reference as used during clinical recordings of evoked brainstem potentials in our laboratory). The click intensities routinely used in these experiments were either $95 \mathrm{~dB}$ or 100 $\mathrm{dB}$ above the reference (140 and $145 \mathrm{~dB}$ SPL). These clicks are within the range used clinically to record brainstem evoked responses.

Peaks on the averaged EMG record will be described with the mean latency preceded by lower case letters (p13, n23 etc), as proposed

\section{$75 \mathrm{~dB}$}
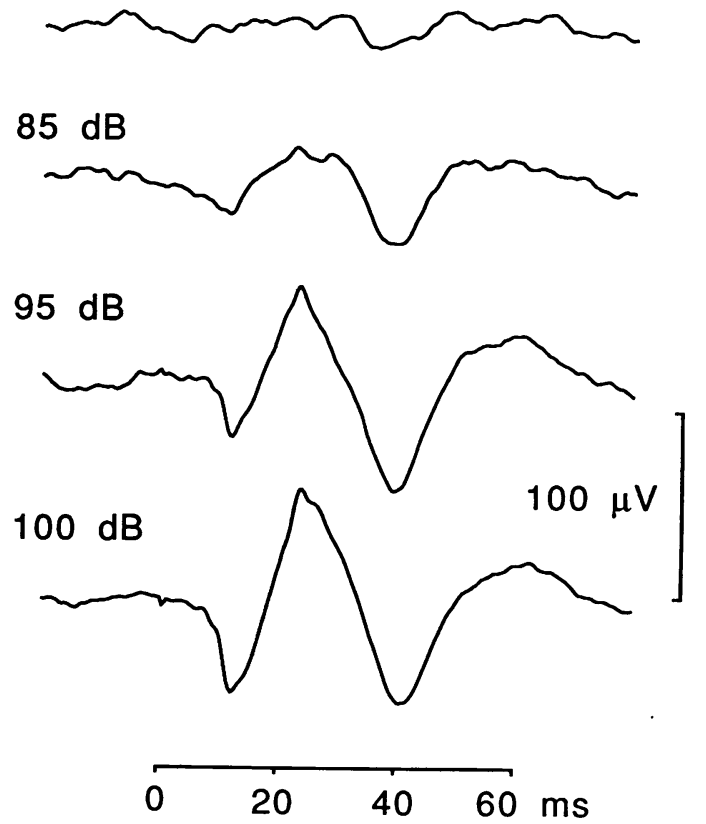

Figure 2 Effect of differing intensities of stimulation in a single subject. $A$ constant target for muscle activation was used for all the trials, resulting in similar levels of mean, prestimulus, rectified EMG (67.0-74.3 $\mu \mathrm{V}$ : averages of rectified EMG not shown). Bilateral clicks were used and averages of 512 presentations made from recording electrodes over the right sternomastoid. All components of the averaged response become progressively larger as the intensity of the clicks is increased. The $p 44$ potential is evident at the lowest intensity used $(75 \mathrm{~dB})$ but the p13-n23 response only appears at the next highest intensity (85 dB). 
Figure 3 Simultaneous averages of rectified and unrectified EMG from over the right sternomastoid of a normal volunteer $(n=512)$. Clicks were presented bilaterally $20 \mathrm{~ms}$ after the onset of the traces. As shown here, the evoked responses were more clearly seen on the unrectified than the rectified average. The mean level of rectified EMG was calculated from the $20 \mathrm{~ms}$ before presentation of the stimul and in this example was $65.7 \mu \mathrm{V}$ (arrow). The calibration bar applies to both traces.
Figure 4 (A) Three separate averages made over the right

sternomastoid in one subject with bilateral clicks of $95 \mathrm{~dB}$ intensity during weak, moderate, and strong contractions of the neck flexors. The clicks were presented $20 \mathrm{~ms}$ after the average began, at the position indicated by the stimulus artifact. The mean level of rectified EMG preceding the three trials was $41 \cdot 3,59 \cdot 2$ and $147.5 \mu \mathrm{V}$ respectively. Size of the averaged potentials was clearly related to the level of tonic muscle contraction during the collection period. (B) (Same subject) peak to peak amplitude of the p13-n23 response to $95 \mathrm{~dB}$ clicks is plotted against the mean level of prestimulus rectified EMG for a total of 19 observations made at different levels of tonic muscle activation. In this and the other volunteers, the relation between the amplitude of the evoked muscle potential and the level of tonic EMG activity was linear. The points include the three

observations shown in $(A)$ (arrows, the leftmost representing the weakest mean contraction level), averages made during maintained head rotation to either the left or right side and also during unilateral stimulation of the ipsilateral (right) ear.
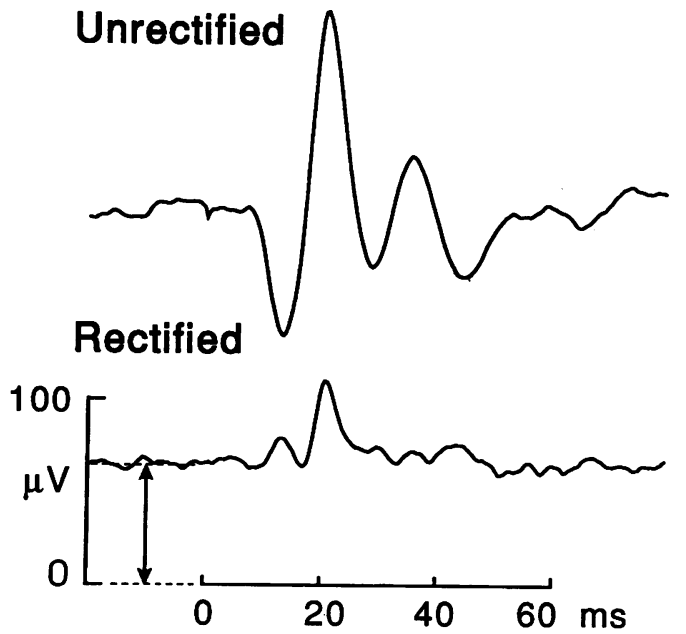

\section{A}
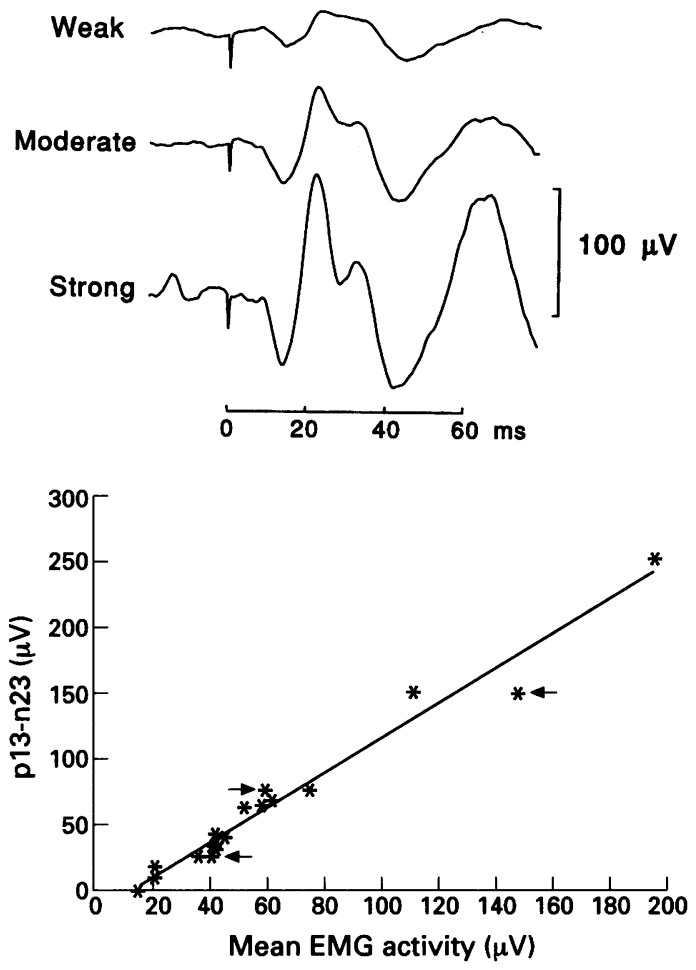

by Yoshie and Okudaira ${ }^{15}$ to distinguish them from conventional, neurally generated evoked potentials. Mean values are given with SDs. Amplitudes were measured either peak to peak or, for a single peak, with reference to the mean level of activity in the $20 \mathrm{~ms}$ preceding the clicks.

\section{Results}

FINDINGS IN NORMAL VOLUNTEERS

All the normal volunteers showed short latency responses to $95 \mathrm{~dB}$ clicks given during tonic neck flexor activation (onset latency $\mathbf{8 \cdot 2}$ $(0.6) \mathrm{ms}$ (right), 8.2 (0.7) $\mathrm{ms}$ (left)). Presented bilaterally, these clicks evoked averaged EMG changes on both the right and left sides, which always began with an initial positive-negative wave (p13-n23: fig 1). Only this early response was present in every subject. Additional potentials often followed (n34, p44) and for any given subject, the evoked response recorded on the right and left sides to bilateral clicks had similar waveforms. The p13-n23 response was present only with high intensity clicks and the threshold varied from 75-85 dB between subjects (fig 2). The threshold for the later responses was below that for the p13-n23 response; as low as $50 \mathrm{~dB}$ in one subject.

\section{Peak latencies}

Latencies on the right and left sides were not significantly different for any of the waveform peaks, but, as noted earlier, only the p13 and n23 potentials were present on both sides in all subjects. For the 10 subjects (20 sides) the mean latency of the first positivity (p13) was $13.3(1.5) \mathrm{ms}$ and that for the first negativity (n23) was $22.6(2.4) \mathrm{ms}$. Six of the 10 subjects had waveforms similar to that illustrated for subject 1 (fig 1 ), whereas in the remainder one or more of the later peaks was absent unilaterally or bilaterally. A second negativity (n34, present in 15 of the 20 sides) occurred at $33.8(2.4) \mathrm{ms}$ and was separated from the
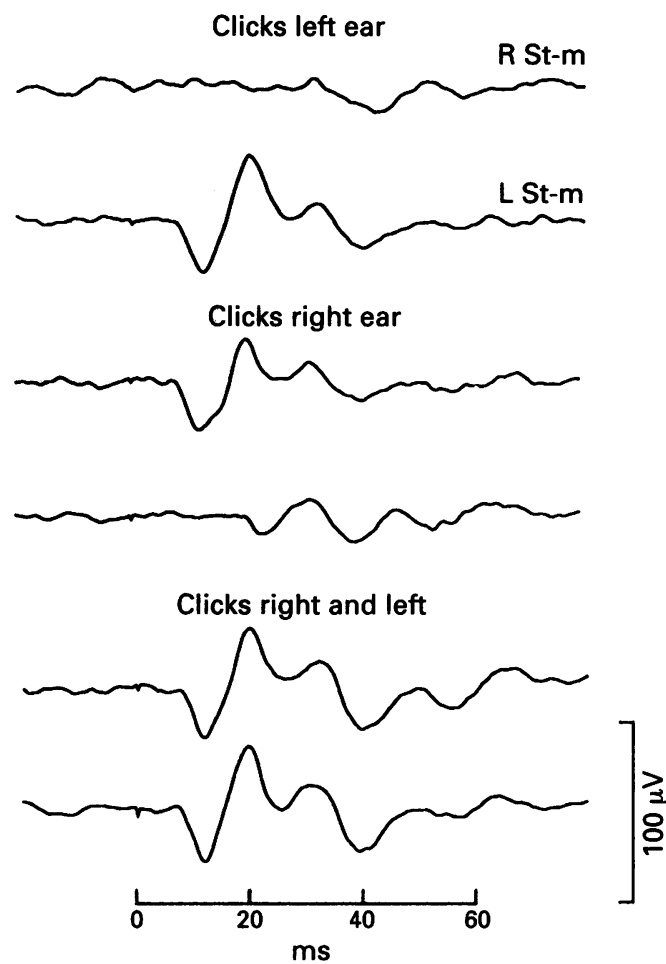

Figure 5 Averaged responses to clicks of $95 \mathrm{~dB}$ intensity applied to each ear in turn. Stimuli applied $20 \mathrm{~ms}$ after each trace starts in all cases. Responses from over the left sternomastoid ( $L S t-m$ ) are shown below those from the right $(R S t-m)$ in each case. With stimulation of the left ear (top pair of traces) the early response occurs only in the recording made over the ipsilateral muscle ( $L S t-m)$.

Similarly, stimulation of the right ear (middle pair) causes an early positive-negative response only in the recording made over the right sternomastoid $(R S t-m)$. All subjects showed an ipsilateral dominance for the origin of p13-n23, indicating that this response is mediated by an ipsilaterally projecting pathway. Unilateral stimulation of each ear does, however, generate longer-latency responses on both sides, indicating that the afferents responsible for these later potentials have bilateral reflex effects. Stimulation of both ears simultaneously (bottom pair) results in p13-n23 responses on both sides, as well as later potentials. 
n23 potential by an inconstant positive going inflexion (present in 13 of the 20 sides) at a mean latency of $28.6(2 \cdot 3) \mathrm{ms}$. A second positivity ( $\mathrm{p} 44$, present in 17 of the 20 sides) occurred at $43 \cdot 7(3 \cdot 6) \mathrm{ms}$.

\section{Effect of tonic EMG activity}

Bickford $e t$ al $^{8}$ reported that the amplitude of the potentials evoked by clicks at the inion was influenced by loading the neck muscles and thereby altering their tonic activation. In the present study, averages of rectified EMG were collected primarily to quantify the level of tonic muscle activation based on the mean rectified value prior to the stimulus (fig 3). Changes in the average rectified EMG after the stimulus were always less pronounced than for the simultaneously acquired unrectified average and amplitudes for the evoked responses were measured from the unrectified averages.

The level of tonic neck muscle EMG was altered between trials by asking the subjects
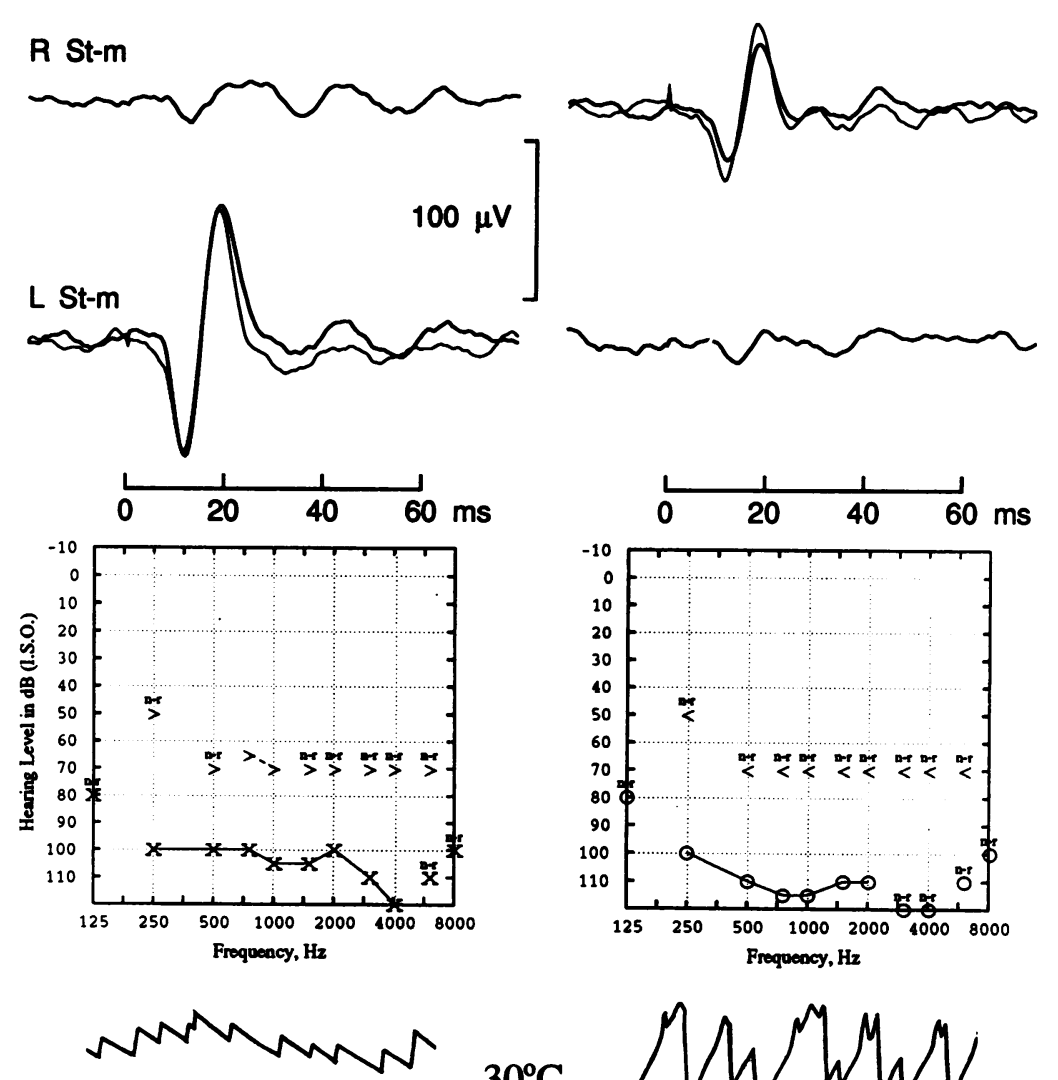

$30^{\circ} \mathrm{C}$
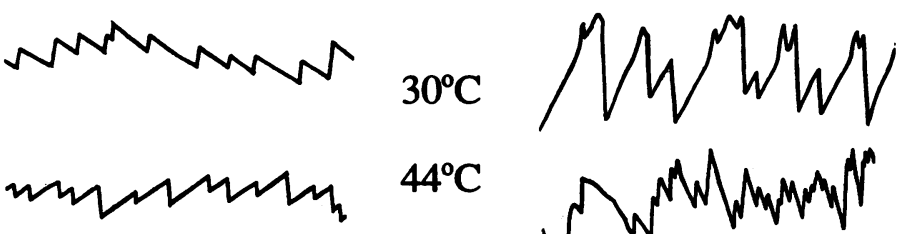

$44^{\circ} \mathrm{C}$

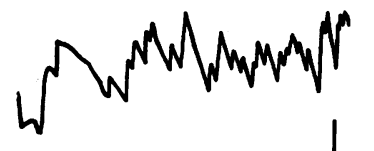

Figure 6 Click-evoked vestibular potentials in response to $100 \mathrm{~dB}$ monaural stimulation in a 14-year-old boy with severe bilateral hearing loss and no vestibular symptoms. The left and right panels relate to the left and right ears. Top: there was an ipsilateral p13-n23 response to stimulating the left ear and the right ear individually (thick lines). The fine lines show the effect of bilateral clicks. Note that the patient was unable to hear the stimulus when presented to the right ear. Middle: audiograms show a greater than $100 \mathrm{~dB}$ (ISO) sensorineural hearing loss in both ears. There was no response (' $n-r$ ') from the patient to either bone conduction $(<$ and $\rangle$ ) or air conduction $(x$ and $o$ ) at maximal output from the stimulator. Bottom: caloric testing showed slightly reduced vestibular responses from the left ear (53\% left canal paresis) and normal responses from the right ear after stimulation with water at $30^{\circ}$ and $44^{\circ} \mathrm{C}$. Upward deflections indicate rightward eye movement, downward deflections indicate lefiward eye movements. Calibration bars are $10^{\circ}$ and $1 \mathrm{~s}$. to press their forehead against the bar or by holding their head rotated to either the left or right side. As reported for the inion response, the amplitude of the averaged potentials after clicks of a fixed intensity was dependent on the level of tonic muscle activation. In all subjects there was a linear relation between the amplitudes of the p13-n23 (and the p44) peak of the evoked response and the mean level of rectified EMG before the stimulus presentation (fig 4). In one subject only the p13-n23 response seemed to saturate at the highest level of EMG: p13-n23 $=374 \mu \mathrm{V}$, mean rectified $E M G=224 \mu \mathrm{V}$, obtained with a near maximal contraction. Values of the squared correlation coefficient for the linear fit between p13-n23 amplitude and mean rectified EMG ranged from 0.81 to 0.99 .

Two values define each linear regression: the intercept on the abscissa (the EMG threshold for a response) and the gradient (the gain of the evoked response). The EMG thresholds derived from the regression of p13-n23 amplitude against rectified EMG were $11.3 \mu \mathrm{V}$ (right) and $11.6 \mu \mathrm{V}$ (left) and did not differ significantly between the two sides $(t=-0 \cdot 12)$. The gain of the response to $95 \mathrm{~dB}$ clicks varied from 0.38 to $3 \cdot 26$, was usually similar for both the right and left sides, and was greater than unity in all but one subject. The regression equation, once defined, can be used to predict the size of the p13-n23 response for any given level of rectified EMG activity - that is, to generate a normalised response. For a mean rectified EMG activity of $60 \mu \mathrm{V}$, the normalised p13-n23 responses to $95 \mathrm{~dB}$ clicks varied between subjects from 18.3 to $137 \cdot 1 \mu \mathrm{V}$ (mean 88.8 (right) and $81 \cdot 8 \mu \mathrm{V}$ (left)).

\section{Effect of stimulus intensity}

All the normal subjects were studied at three to five different stimulus intensities above threshold for their p13-n23 response, including $100 \mathrm{~dB}$ clicks in five subjects. Each of the runs was performed with a constant EMG target to minimise changes in tonic activity. The p13-n23 amplitude increased with increasing stimulus intensity and on average the p13-n23 response to $100 \mathrm{~dB}$ clicks was $36 \%$ larger than that to $95 \mathrm{~dB}$ clicks (fig 2). The effect of suprathreshold click intensity seemed to be linear (intensity measured in dB) with squared correlation coefficients 0.78 or greater in all but the right side of one subject. Thresholds for the p13-n23 response deduced from the intercept of this relation varied from 64 to $80 \mathrm{~dB}$.

\section{Laterality of responses}

A clear difference between the origin of the early (p13-n23) response and of the later potentials was seen after unilateral ear stimulation. With clicks applied to only one ear, the p13-n23 potential was always larger on the ipsilateral side (fig 5 ). In several subjects this ipsilateral predominance was complete and there was no detectable crossed p13-n23 response. On average, the ipsilateral p13-n23 response evoked by unilateral stimulation was 
$98 \%$ ( $91 \%$ for responses recorded on the right side, $104 \%$ for responses recorded from the left) of that evoked by bilateral stimulation, whereas the crossed response to unilateral ear stimulation was on average only $12 \%$ of that evoked by bilateral ear stimulation ( $10 \%$ recorded right; $14 \%$ left). By contrast with this finding, the later potentials always occurred bilaterally even with unilateral stimulation, and the effects of bilateral stimulation were additive: thus the amplitude of the p44 response evoked with unilateral ear stimulation was on average about half the size of that recorded at the same site with bilateral stimuli (mean $55 \%$ of the bilateral response amplitude).

Occasionally, the absence of a p13-n23 response on the contralateral side with unilateral stimulation revealed the presence of a short latency negativity, starting slightly later than the usual $\mathrm{p} 13$ potential. This seemed to habituate between trials and was not studied in detail.
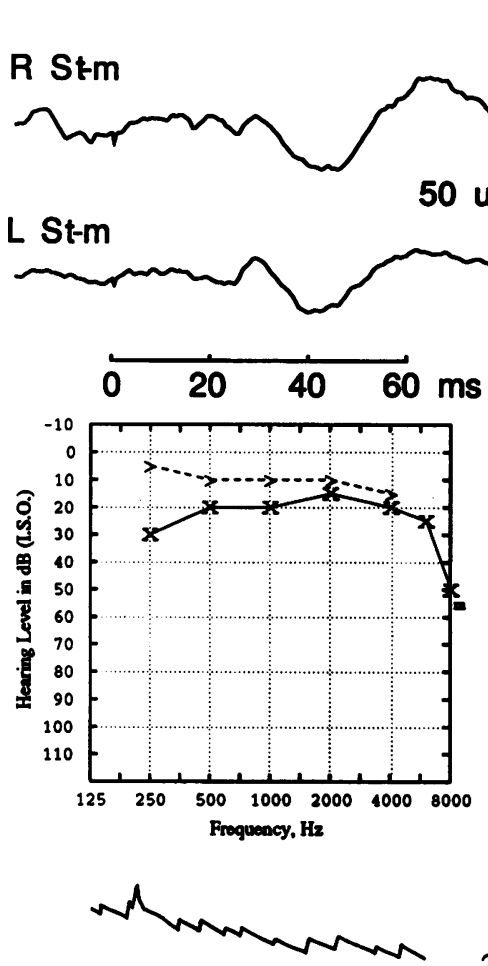

$30^{\circ} \mathrm{C}$
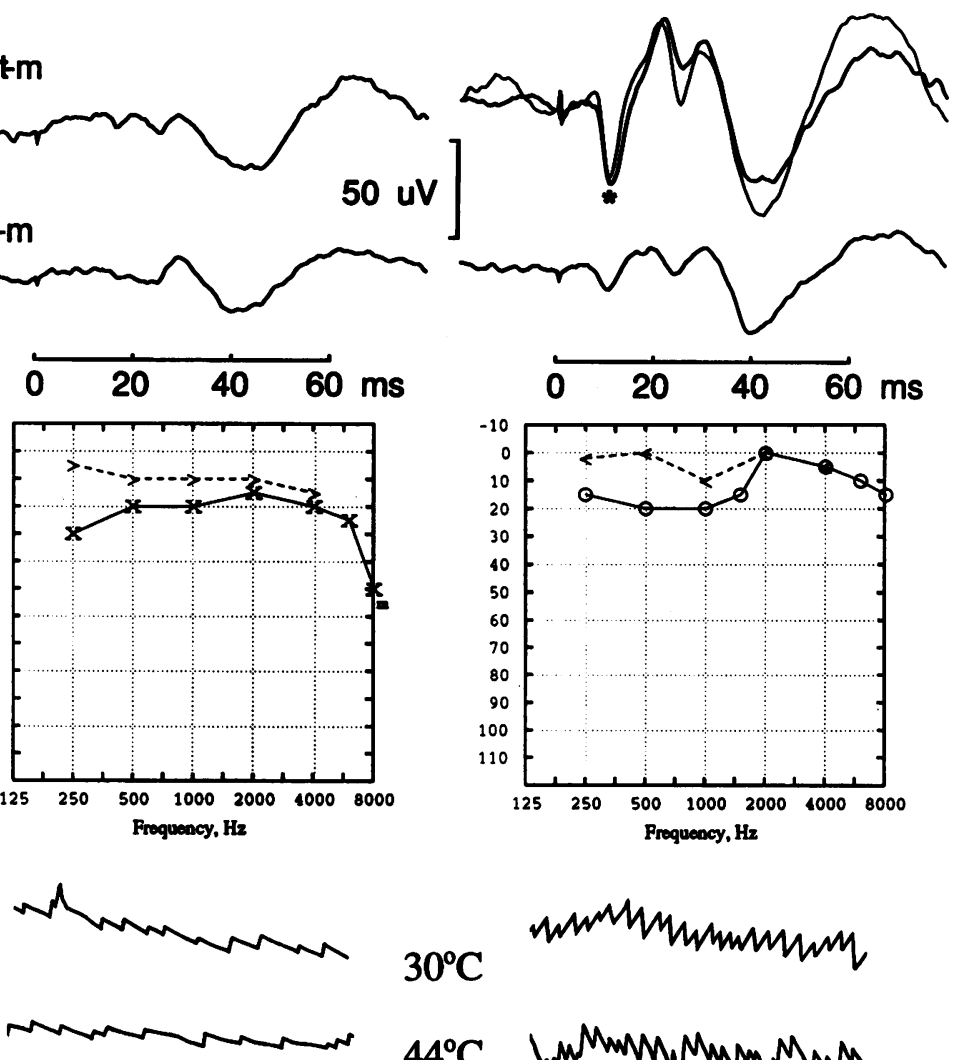

$44^{\circ} \mathrm{C}$

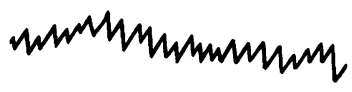

$0^{\circ} \mathrm{C}$
Additional findings in normal subjects

In two subjects, control studies showed that there was no response to unilateral clicks presented when the external ear canal was plugged and that the response returned completely when a small hole was made in the plug. The responses of one subject were profoundly attenuated when tested during an upper respiratory tract infection with sinusitis but returned to normal on recovery. In another subject a Valsalva manouevre performed against an occluded nose also attenuated the response (not illustrated).

\section{RECORDINGS FROM PATIENTS WITH LESIONS OF} THE INNER EAR

Selective sensorineural deafness

Three patients who were found to have pronounced sensorineural deafness in one or both ears were studied. They had no symptoms or signs of vestibular disease and caloric testing was normal. Pure tone audiograms showed perceptual thresholds higher than 80 $\mathrm{dB}$ (ISO) at all frequencies for each patient's most severely affected ear. All three patients had p13-n23 responses after stimulation of their affected ear (fig 6).

\section{Selective vestibular nerve section}

Five patients who had each undergone selective surgical section of the vestibular nerve as treatment for intractable vertigo ${ }^{16}$ were studied. One man was studied before and three months after selective nerve section (also reported by Colebatch and Halmagyi ${ }^{14}$ ) and the four women had undergone the operation from one to seven years previously. None of the five had severe hearing loss in either ear. The findings in four of the patients were the same: the p13-n23 response was obtained only from the ear innervated by the intact vestibular nerve (fig 7) although longer latency potentials were still obtainable from the operated ear. The ipsilateral p13-n23 response previously present was selectively abolished postoperatively in the man (the remaining woman, known to have otosclerosis, had no detectable p13-n23 after stimulation of either ear at $100 \mathrm{~dB}$ intensity).

\section{Discussion}

NATURE AND ORIGIN OF THE RESPONSE

These experiments have demonstrated that brief, intense clicks cause reproducible, shortlatency alterations in EMG activity in the anterior neck muscles of normal subjects. Faithful transmission of the clicks to the inner ear was required: conductive hearing loss either from occlusion of the external ear or due to alterations within the middle ear, attenuated or abolished the evoked responses. In normal volunteers the evoked response comprised two distinct components. The first, a biphasic positive-negative wave (p13n23), was generated predominantly by afferents originating ipsilaterally and depended on the integrity of the labyrinth and the vestibular division of the eighth nerve. This initial response always had a high stimulus thresh- 
old. The later components of the averaged response, not always present in normal subjects, usually began with a negativity and were generated by afferents originating from both ears. These potentials were present when the cochlea and cochlear component of the eighth nerve alone were intact. This evidence indicates that the p13-n23 response originates from afferents travelling within the vestibular nerve whereas the later potentials probably arise from cochlear afferents. Both Ruhm et al $^{17}$ and Yoshie and Okudaira ${ }^{15}$ proposed that both cochlear generated and vestibular generated myogenic reflexes might exist but implicitly assumed that at each specific recording site, one or other mechanism was responsible. Our findings that both vestibular and cochlear generated effects underlie the evoked response, if also true of the effects of clicks on posterior neck muscles, would largely resolve the apparent contradictory reports as to the origin of the inion response.

Sound-evoked myogenic responses in cranial muscles-the postauricular response ${ }^{18}$ and the acoustic jaw reflex ${ }^{11}$-are well described. Both these reflexes seem to depend on cochlear afferents and are evoked bilaterally after stimulation of one ear. Indeed, this may be a general feature of all reflexes generated by cochlear afferents, as auditory pathways in the brainstem are characterised by well developed commissural connections at multiple levels. ${ }^{1}$ The second part of the averaged response described here was distributed bilaterally and seems attributable to cochlear activation. This cochlear dependent response might be thought to be related to the startle reflex, a normal response to any unexpected, intense stimulus. Physiological startle to sound is characterised by rapid habituation, a prolonged refractory period, and a much longer latency than the averaged responses documented here ${ }^{19}$ and thus is best regarded as a separate phenomenon.

The p13-n23 potential had characteristics that distinguish it from the later potentials and seems to originate from activation of the ipsilateral labyrinth. In certain disorders affecting the middle and inner ear, vestibular activation can occur with sound-the Tullio phenomenon. ${ }^{720}$ Vestibular stimulation by sound is not confined solely to pathological states but the intensities needed to excite the normal vestibular apparatus are louder than normally encountered in everyday life. In monkeys, single unit recordings from the vestibular nerve have shown that saccular afferents have the lowest threshold to acoustic stimulation (typically 106-110 dB SPL for phase locking to low frequencies) an effect that is probably the result of direct excitation of hair cells. ${ }^{21}$ Békésy $^{22}$ reported that with continuous sound in the range of $500-1000$ dynes $/ \mathrm{cm}^{2}$ (128-134 dB SPL or $83-89 \mathrm{~dB}$ relative to our reference), he could induce sensations of movement and involuntary head movements in humans. Finally, in guinea pigs, saccular afferents are excited by clicks with the same duration and relative intensity as those used here. ${ }^{23}$
CENTRAL PATHWAY OF THE VESTIBULAR DEPENDENT RESPONSE

The latency of the earliest responses recorded here implies a rapidly conducting, disynaptic pathway. The observed latency represents the sum of the delays due to activation of receptors, conduction to the brainstem, central conduction from the pons to the motor nucleus of the sternomastoid muscle, and peripheral conduction from the motor nucleus to the muscle itself. In guinea pigs, a click-evoked volley in the inferior vestibular nerve appears at a latency of less than $0.5 \mathrm{~ms}$ (fig 2 of Didier and Cazals ${ }^{23}$ ) and electrical stimulation of the vestibular nerve in cats evokes spike potentials in the axons entering the brainstem at a mean latency of $0.66 \mathrm{~ms}^{24}$ The spectrum of vestibular nerve fibre diameters is similar in monkeys and guinea pig, ${ }^{25}$ and, allowing for the greater conduction distance in humans, a peripheral conduction delay to the brainstem of at least $1 \mathrm{~ms}$ seems appropriate. The fibres innervating the sternomastoid muscle originate from the second to the fifth cervical spinal segments and ascend through the foramen magnum and then leave the cranium through the jugular foramen. ${ }^{1}$ Brown et al ${ }^{26}$ used magnetic stimulation over the spinal roots, and estimated peripheral conduction delay to the sternomastoid muscle to be $5.2 \mathrm{~ms}$. Several subjects in the present series had latencies to the onset of the p13 potential of $8 \mathrm{~ms}$ or less. With this figure, only $1.8 \mathrm{~ms}$ remains for central conduction time. As nearly all primary vestibular afferents terminate in the vestibular nuclei, ${ }^{1}$ central conduction time must include at least two synaptic delays - the second being at the level of the sternomastoid motor nucleus. If $0.6 \mathrm{~ms}$ is allowed for each synapse, the central pathway cannot include more than these two synapses and the conduction delay of the vestibulocollic projection fibres must be of the order of $0.5 \mathrm{~ms}$. Taking the distance from mid-pons to mid-C2 as $5 \mathrm{~cm},{ }^{27}$ a $0.5 \mathrm{~ms}$ conduction delay implies that the second order neurons have a conduction velocity of about $100 \mathrm{~m} / \mathrm{s}$. These features are consistent with previously reported characteristics of vestibulospinal projections. Monosynaptic effects of vestibulospinal fibres, both excitatory and inhibitory, are most powerful in neck motoneurons ${ }^{28}$ and most vestibulocollic neurons are monosynaptically excited from the ipsilateral labyrinth. ${ }^{29}$ The lateral vestibulospinal tract (LVST) originates predominantly from the lateral (Deiter's) nucleus and projects ipsilaterally. Second order neurons, monosynaptically excited from the ipsilateral vestibular nerve, are particularly common amongst ventral LVST neurons projecting to the neck and the modal conduction velocity of the LVST is $90 \mathrm{~m} / \mathrm{s} .{ }^{30}$ As saccular afferents terminate in the LVST, ${ }^{31}$ at least one potential pathway has been shown by which the effects shown here could reach the sternomastoid nucleus.

CHANGES WITH THE LEVEL OF EMG ACTIVITY The nature of the click stimulus allowed study of the effects of tonic neck activation 
on the size of the reflex while keeping the afferent volley constant. Both the vestibular dependent and cochlear dependent components increased linearly with increasing tonic muscle activation. This relation cannot be due to changes in the discharge of cutaneous pressure receptors excited by increasing forehead pressure on the head restraint because it was also true when the head was free and held rotated (little habituation to the stimulus could have occurred during the course of the experiment, otherwise systematic deviations from the linear relation would have been apparent). The long-latency stretch reflex has the property of scaling in size in proportion to the level of tonic muscle activity, a feature that is of particular importance for a reflex, the effectiveness of which must be matched to the voluntary task being undertaken. ${ }^{32}$ Although Marsden et al ${ }^{32}$ initially postulated a supraspinal mechanism for this scaling, a similar relation has since been shown for the segmental stretch reflex. ${ }^{33}$ Our finding that both the vestibular-dependent and cochlear-dependent components of the evoked response scale linearly with the level of tonic EMG suggests that the mechanism lies at the level common to both, the sternomastoid motor nucleus. Matthews ${ }^{34}$ has argued that scaling of both the excitatory and inhibitory effects of Ia afferent discharge is likely to be an intrinsic feature of the motoneuronal pool, given, inter alia, that the reflex effects are distributed throughout the motoneuronal pool and that their nature does not alter between motoneurons of different recruitment thresholds. Clearly, the conditions necessary for such a relation are not unique to proprioceptive reflexes.

\section{PHYSIOLOGICAL ROLE OF VESTIBULOCOLLIC REFLEXES}

Vestibulospinal influences contribute both to the posture adopted by an organism and to maintenance of that posture despite perturbations. This role is emphasised by the commonly used definition of the (dynamic) vestibulocollic reflex by its ability to stabilise the position of the head in absolute space in response to unpredictable displacements. Investigations of this property have confirmed a contribution from the vestibular apparatus in stabilising head position but one that has modest gain and which is dependent on subject instruction or set. ${ }^{35-37}$ In addition to any such role, experimental evidence suggests that vestibular influences may in part determine the posture of the head adopted both by animals and humans. Decerebrated animals tend to maintain their heads vertical independent of the position of the rest of the body. ${ }^{38}$ Acute unilateral loss of vestibular input in humans is associated with tonic lateral deviation of the head and eyes to the same side, ${ }^{39} 40$ usually transient, but occasionally followed by permanent abnormalities of head posture. ${ }^{41}$ The vestibulocollic reflex demonstrated in the present experiments occurs at short latency and has an amplitude that scales with the mean level of EMG activity. It is thus well suited to modifying or fine tuning voluntary motor commands directed to neck muscles. It is also a simple new method of exploring a vestibulocollic pathway in intact humans.

JGC held a CJ Martin Fellowship from the National Health and Medical Research Council of Australia. This study was also supported by a grant from the Clive and Vera Ramaciotti Foundations. We thank Professors JW Lance and D Burke for their helpful criticism.

1 Brodal A. Neurological anatomy. 3rd edn. New York: Oxford University Press, 1981.

2 Baloh RW, Honrubia V. Clinical neurophysiology of the vestibular system. Philadelphia: FA Davis, 1979:132.

3 Brandt T. Man in motion: historical and clinical aspects of vestibular function. Brain 1991;114:2159-74.

4 Melvill Jones G, Watt DGD. Muscular control of landing from unexpected falls in man. $f$ Physiol 1971;219: 729-37.

5 Greenwood R, Hopkins A. Muscle responses during sudden falls in man. F Physiol 1976;254:507-18.

6 Watt DGD. Responses of cats to sudden falls: an otolithoriginating reflex assisting landing. $\mathcal{f}$ Neurophysiol 1976; 39:257-65.

7 Dieterich M, Brandt Th, Fries W. Otolith function in man. Brain 1989;112:1377-92.

8 Bickford RG, Jacobson JL, Cody DTR. Nature of averaged evoked potentials to sound and other stimuli in man. Ann N Y Acad Sci 1964;112:204-23.

9 Cody DTR, Bickford RG. Averaged evoked myogenic responses in normal man. Laryngoscope 1969;79:400-16.

10 Townsend GL, Cody DTR. The averaged inion response evoked by acoustic stimulation: its relation to the saccule. Ann Otol Rhinol Laryngol 1971;80:121-31.

11 Meier-Ewert K, Gleitsmann K, Reiter F. Acoustic jaw reflex in man: its relationship to other brain-stem and microreflexes. Electroencephalogr Clin Neurophysiol 1974; 36:629-37.

12 Cody DTR, Jacobson J, Walker JC, Bickford RG. Averaged evoked myogenic potentials to sound in man Ann Otol Rhinol Laryngol 1964;80:121-31.

13 Douek E. Auditory myogenic responses. In: Beagley HA ed. Audiology and audiological medicine. Oxford: Oxford University Press, 1981:769-80.

14 Colebatch JG, Halmagyi GM. Vestibular evoked potentials in human neck muscles before and after unilatera vestibular deafferentation. Neurology 1992;42:1635-6.

15 Yoshie N, Okudaira T. Myogenic evoked potential responses to clicks in man. Acta Otolaryngol (Stockh) 1969;252 (suppl):89-103.

16 Silverstein $H$, Norell $H$, Rosenberg $S$. An evolution in approach in vestibular neurectomy. Otolaryngol Head Neck Surg 1990;102:374-81.

17 Ruhm H, Walker E, Flanigin H. Acoustically-evoked potentials in man: mediation of early components. Laryngoscope 1967;77:806-22.

18 Kiang NY. Postauricular electric response to acoustic stimuli in humans. Q Prog Rep Res Lab Elec MIT 1963 68:218-25.

19 Brown P, Rothwell JC, Thompson PD, Britton TC, Day $B L$ Marsden CD. New observations on the normal auditory startle reflex in man. Brain 1991;114: 1891-902.

20 Ishizaki H, Pyykko I, Aalto H, Starck J. Tullio phenomenon and postural stability: experimental study in normal subjects and patients with vertigo. Ann Otol Rhinol Laryngol 1991;100:976-83.

21 Young ED, Fernandez C, Goldberg JM. Responses of squirrel monkey vestibular neurons to audio-frequency squirrel monkey vestibular neurons to audio-frequency sound and head

22 von Békésy G. Uber akustische Reizung des Vestibularapparates. Pfügers Arch 1935;236:59-76.

23 Didier A, Cazals Y. Acoustic responses recorded from the saccular bundle on the eighth nerve of the guinea pig. Hear Res 1989;37:123-8.

24 Precht $W$, Shimazu $H$. Functional connections of tonic and kinetic vestibular neurons with primary vestibula afferents. $\mp$ Neurophysiol 1965;28:1014-28.

25 Gacek RR, Rasmussen GL. Fiber analysis of the stereoacoustic nerve of guinea pig, cat, and monkey. Anat Rec 1961;139:455-63.

26 Brown P, Thompson PD, Rothwell JC, Day BL, Marsden CD. Axial myoclonus of propriospinal origin. Brain 1991;114:197-214.

27 Koritke JG, Sick H. Atlas of sectional human anatomy. 2nd edn. Baltimore: Urban and Schwarzenberg, 1988:24-5.

28 Wilson VJ, Yoshida M. Comparison of effects of stimulation of Deiters' nucleus and medial longitudinal fasciculus on neck, forelimb, and hindlimb motoneurons. lus on neck, forelimb, and

29 Rapoport S, Susswein A, Uchino Y, Wilson VJ. Properties of vestibular neurones projecting to neck segments of of vestibular neurones projecting to neck segm

30 Wilson VJ, Peterson BW (1981) Vestibulospinal and reticulospinal systems. In: Brooks VB, ed. Handbook of physiology, section 1: motor control. Bethesda, MD American Physiological Society, 1981;2:667-702. 
31 Didier A, Cazals Y, Aurousseau C. Brainstem connections of the anterior and posterior parts of the saccule of the guinea pig. Acta Otolarmol (Stockh) 1987:104.385-91.

32 Marsden CD, Merton PA, Morton HB. Servo action in the human thumb. $₹$ Physiol 1976;257:1-44.

33 Marsden CD, Rothwell JC, Day BL. Long-latency automatic responses to muscle stretch in man: origin and matic responses to muscle stretch in
function. Adv Neurol 1983;39:509-39.

34 Matthews PBC. Observations on the automatic compensation of reflex gain on varying the pre-existing level of motor discharge in man. F Physiol 1986;374:73-90.

35 Guitton D, Kearney RE, Werely N, Peterson BW. Visual, vestibular and voluntary contributions to human head stabilization. Exp Brain Res 1986;64:59-69.

36 Gresty $M$ (1987) Stability of the head: studies in normal subjects and in patients with labyrinthine disease, head tremor, and dystonia. Mov Disord 1987; 2:165-85.
37 Bronstein AM. Evidence for a vestibular input contributing to dynamic head stabilization in man. Acta Otolaryngol (Stockh) 1988;105:1-6.

38 Gernandt BE. Vestibular mechanisms. In: Magoun HW, ed. Handbook of physiology, section 1: neurophysiology. Washington: American Physiological Society 1959;1: 549-64.

39 Holmes G. Introduction to clinical neurology. Edinburgh: Livingstone, 1952:139-40.

40 Halmagyi GM, Curthoys IS, Brandt $T$, Dieterich $M$ Ocular tilt reaction: clinical sign of vestibular lesion. Acta Otolaryngol (Stockh) 1991;481 (suppl):47-50.

41 Bronstein AM, Rudge $P$. The vestibular system in abnor$\mathrm{mal}$ head postures and spasmodic torticollis. Adv Neurol 1988;50:493-500.

42 Smith PF, Curthoys IS. Mechanisms of recovery following unilateral labyrinthectomy: a review. Brain Res Rev $1989 ; 14: 155-80$

\section{Von Monakow and Diaschisis}

For much of the 19th century, clinical localisation rested on the belief that geometric localisation produced signs dependent on functions derived from that focal area. In 1967 Geschwind's “disconnection syndromes" highlighted the phenomenology of dysfunction at a distance. Geschwind's notions, now accepted, hark back to the work of Constantin Von Monakow (1853-1930). ${ }^{1}$ He introduced the word "diaschisis" to indicate loss of function and signs, anatomically removed from the lesion-the equivalent of Geschwind's disconnection. Von Gudden had shown him atrophy of the superior colliculus in a rabbit after removal of the contralateral eye at birth. In 1879 he removed the occipital lobes in two newborn rabbits and one year later noticed complete degeneration of the lateral geniculate nuclei.

Von Monakow described diaschisis cortico-spinalisimpaired function of spinal motor neurons due to cortical motor lesions; diaschisis associativa-cortical dysfunction due to lesions of connected areas within one hemisphere, and diaschisis commisuralis-cortical dysfunction due to lesions of the interconnected contralateral hemisphere.

He refuted the purist's notions of localisation:

The generally accepted theory according to which aphasia, agnosia, apraxia etc. are due to destruction of narrowly circumscribed appropriate praxia, gnosia, and phasia centres, must be finally discarded on the basis of more recent clinical and anatomical studies. It is just in the case of these focal symptoms that the concept of complicated dynamic disorders in the whole cortex becomes indispensable.

The verification of Von Monakow's postulates has been achieved by oxygen and glucose uptake studies, and by disordered blood flow and electrophysiology in distant sites.

The syndrome of anterior choroidal thrombosis causing contralateral hemiplegia, hemianaesthesia and hemianopia is referred to as Von Monakow's syndrome. He also wrote extensively on the cortex, microcephaly, anencephaly, and aneurysm of the vertebral artery.

Born the son of a Russian nobleman, his family fled the Franco-Prussian war, settling in Dresden, then Paris, and Zurich where they were naturalised in 1869. A huge, bearded figure with a shrill voice, he was noted, in youth, for his boisterous, eccentric behaviour. Later, he eschewed faculty meetings, and was devoted to his family and books. His famous Gehirnpathologie contained over 3000 references that he had collected personally. In later life he published three lectures, entitled: Emotions, morality and the brain. He died of "retention-uraemia" aged 77 while writing on "The value of life".

JMS PEARCE 304 Beverley Road Anlaby, Hull

1 Von Monakow C. Die lokalisation im Grosshim und der Abbau der Funktion durch kortikale Herde. Wiesbaden: Bergmann 1914. Transl. G Harris In: Pribam KH, ed. Brain and Behavior I: Mood States and Mind. Baltimore: Penguin 1969:27-36. 